\title{
An integrated approach with homeopathic medicine and electro-acupuncture in anaesthesiology during breast cancer surgery: Case reports
}

\author{
Bosco $\mathrm{F}^{1 *}$, Cidin $\mathrm{S}^{1}$, Maceri $\mathrm{F}^{1}$, Ghilli $\mathrm{M}^{2}$, Roncella $\mathrm{M}^{2}$, De Simone $\mathrm{L}^{1}$ \\ S.D. Anaesthesia and Intensive Care MiSC AOUP Complementary Medicine Oncology Integrated Breast Unit, University Hospital Trust of \\ Pisa, Via Roma 67, 56126, Pisa, Italy \\ ${ }^{2}$ Breast Cancer Surgical Unit, University Hospital Trust of Pisa, Via Roma 67, 56126, Pisa, Italy
}

\section{Key Words}

Anaesthesiology; Breast cancer; Electro-acupuncture; Homeopathy; Multidisciplinary approach

\begin{abstract}
This study investigates the effect of a combination of homeopathic medicine and electro- acupuncture in two patients with breast cancer and severe liver disease who could not receive standard anaesthesia therapy due to liver problems. Specifically, measurable and quantifiable parameters were used to evaluate whether an integrated approach-consisting of electro- acupuncture and a homeopathic medicine diluted above Avogadro's limit (that is, above a potency of $12 \mathrm{CH}$ ) during the pre-surgical, surgical and post-surgical phases-can improve general well-being of a patient undergoing breast cancer surgery. In breast cancer surgery, we employed an integrated approach consisting of induction with hypnotics and muscle relaxants, followed by maintenance with anaesthetic gas, combined with a homeopathic treatment (Arnica montana $15 \mathrm{CH}$ and Apis mellifica $15 \mathrm{CH}$ ) before and after surgery and an electro- acupuncture treatment performed in the pre-
\end{abstract}

Received: Sep 12, 2017 Reviewed: Mar 20, 2018 Accepted: May 17, 2018

(c) This is an Open-Access article distributed under the terms of the Creative Common Attribution Non-Commercial License (http://creativecommons.org/licenses/by-nc/4.0/) which permits unrestricted noncommercial use, distribution, and reproduction in any medium, provided the original work is properly cited.

(@) This paper meets the requirements of KS X ISO 9706, ISO 9706-1994 and ANSI/NISO Z39.48-1992 (Permanence of Paper). and post-surgical phases without any analgesic/pain relieving medications. Both of the patients treated with the integrated approach improved their overall condition without need for other common pain relieving medicines. Additionally, thanks to their rapid awakening, the patients were not relocated to a protected area and the hospitalization was shorter. A multidisciplinary approach incorporating homeopathic medicine and electro-acupuncture can be a solution for patients who need or ask about a different and/or safer alternative to the standard treatment. This approach can offer a safe, much less expensive, non-invasive and viable alternative for such cases. Moreover it can be useful for an opioids free anesthesia.

\section{Introduction}

Pain during surgery is not easy to treatl and, often, a multidisciplinary approach that integrates different treatments is useful to improve the well-being of patients undergoing surgery. Among these, acupuncture and homeopathy play an important role in today's multidisciplinary approach for the treatment of pain during surgery2-5. According to this 6-10, we employed electro-acupuncture and homeopathic medicines in association with traditional anaesthesiology for control of analgesia. Acupuncture is widespread in surgery 11-12 Analgesia with electro-acupuncture (EAP) is obtained through an elevation of the threshold of pain and an in-
"Corresponding Author

S.D. Anaesthesia and Intensive Care MiSC AOUP Complementary Medicine Oncology Integrated Breast Unit, University Hospital Trust of Pisa, Via Roma 67, 56126, Pisa, Italy Tel. +393343292716 email: filbosco@gmail.com 
creased level of morphinomimetic endogenous substances 13-15.

The equipment used for EAP consists of simple direct-current (DC) impulse generators, provided with oscillator circuits that generate the two types of waveforms prevalently used-- square waves and peaked waves--with frequencies (cycles per second) that can range from a minimum of 0.5 to a maximum of 5000 Hertz. All the waveforms and frequencies generally have the effect of promoting circulation of Qi and blood, and thereby provide relief for many symptoms, in particular for pain. The current output by the devices is relatively low, from 10 to 80 milliamperes (mA), depending on the setting used, while the voltage can range from 40 to 80 Volts, and the frequency from a minimum of 1 up to $100 \mathrm{~Hz}$. The mode of output can be continuous, intermittent, or in pulse trains. Generally the standard stimulators do not allow modification of the shape of the wave itself, which is always biphasic square (positive pulse with negative spike and pulse width of $0.2 / 0.4$ milliseconds), considered to be the best for analgesial6.

Homeopathy has been found to be helpful before, during and after surgery 3, 17. Among homeopathic medicines, Arnica montana and Apis mellifica are the ones most widely used in a surgical setting $3,4,17-20$. Arnica montana is commonly used to treat inflammation, wounds, hematoma, contusion and pain, by virtue of its chemical composition21. Depending on the quality of the raw material, the active compounds identified in Arnica m. are above all sesquiterpene lactones22. There is some experimental evidence in vivo of an anti- inflammatory action of Arnica montana administered as an ethanolic extract23 or at the 6th centesimal homeopathic dilution (6CH) 24. A French recommendation includes it among the suggested medicines (Grade Ba - Recommendations level based on RCT studies) 25. Recently, Bellavite et al. have provided new insights into how Arnica m. acts in tissue healing, and identified extracellular matrix regulation by macrophages as a therapeutic target 26 . In vitro studies show activity of Apis mellifica on regulatory processes of inflammation 27, and it has also proven useful in the treatment of pain related to lactation 20. Integrated anaesthesia could be useful in those cases where the patient refuses the standard treatments or "expresses a wish" for alternative ones, as well as in cases where the standard treatments might be contraindicated. Moreover, other clinicians, such as hepatologists, may suggest this type of anaesthesia to reduce the therapeutic load and stress linked to surgery. This has already occurred in our reality.

The aim of this work is to evaluate, through easy and practical measures, whether the use of acupuncture and homeopathy improves the condition of the patient during the surgical procedure. It additionally evaluates whether these can offer an alternative-where needed--to the conventional medicines used for treatment of pain, avoiding their known adverse effects and impact on the immune system.

\section{Case reports}

The multidisciplinary approach represents an opportunity, especially in cases where the patient refuses the standard drug (such as opioids) or explicitly asks for an alternative one, or in situations where the standard drug is contraindicated. Our first experiences were conducted in the surgical unit of the Senology Department at the Pisa University Hospital, where the integration of complementary medicines into the common service provided to patients has been ongoing for several years. This approach was prompted by direct enquiries from patients, and specific requests from hepatologists who had observed some cases with organic disorders, and so inquired into the possibility of some alternative method.

We began with a patient who asked, during the pre-surgical anaesthesiologist visit, about the possibility of avoiding opioid drugs during anaesthesiology and in the post-surgical phase. The planned surgical procedure was a quadrantectomy for breast cancer combined with reductive mastoplasty and bilateral implant removal. The breast surgery, lasting 2 hours and 40 minutes, was conducted with an integrated approach consisting of induction with hypnotics and muscle relaxants, and maintenance with anaesthetic gas. All these phases were accompanied by a homeopathic treatment before and after the surgery and an electro-acupuncture treatment performed in the pre- and post-surgical phases without any analgesic/pain medications. The awakening and post-surgical phases were good: the patient quickly regained consciousness. This first positive experience with this integrated approach encouraged us to repeat, modify and improve the technique.

Accordingly, in this article we illustrate two similar cases. The two patients were the same age (74 years) and both suffered from an advanced stage of a liver disease, with all the complications which that entails.

\section{CASE 1}

The patient was a woman 74 years old, weight $58 \mathrm{~kg}$, height $160 \mathrm{~cm}$ with a diagnosis of bilateral breast cancer.

From the pathological anamnesis, we observed a complicate situation: previous episodes of atrial paroxysmal fibrillation, hepatic cirrhosis from a previous infection by HCV, severe portal hypertension, oesophageal varices and portal thrombosis of the main branch and of the portal trunk, hypersplenism syndrome. From 2010 to 2012 she suffered from continuous events of pancreatitis caused by biliary lithiasis and from 2015 she reported 2 needles of hepatic cancer that couldn't be treated with surgery. In June 2015 she underwent banding of the esophageal varices.

Prior to surgery, a complete hepatic evaluation was performed. We observed good clinical and functional compensation, but also an increased risk of functional hepatic failure. The main intra- and post-surgical risk of hepatic injury is that correlated to possible prolonged hypotension or to pharmacological toxicity.

The chosen surgical procedure for the patient was a quadrantectomy of the right superior breast quadrant, external-superior left quadrantectomy and a bilateral biopsy of the sentinel lymph node. In addition, the evening before the surgery, the patient received a treatment with electro-acupuncture. We used sterile, single-use bimetallic needles with diameter $0.25 \mathrm{~mm}$ and length $25 \mathrm{~mm}$ 
(Hwato). The insertion technique was without guide tube. The insertion depth of each needle was determined separately for each point, based on the reference standards for that point. The electro-stimulation was applied using an E600HAN instrument with dual power supply (mains power / battery) and a digital display that shows the duration of the treatment, and the frequency, intensity and type of waveform used. The available electro-acupuncture settings comprised: A 6 channel output, with symmetric biphasic square waveform with negative spike (Mod. Han); Waveform types: continuous, intermittent, ripple, dense-sparse, modulated, Han mode; Intensity (wave amplitude) of each individual output; Pulse rate from 1 to $500 \mathrm{~Hz}$; Pulse width from 50 to $400 \mu$ s; Programmable countdown timer from 1 to 60 minutes. We configured the treatment with the following parameters:

- Wave type: modulated

- Frequencies: from 23 to $70 \mathrm{~Hz}$.

- Pulse width: 150 microseconds.

- Time: 20 minutes

The selection of the insertion points was fundamental: In the case of postoperative pain treatment, there exist surgical analgesia protocols systematically used by various Chinese hospitals (Nguyen et al, 1984). According to these protocols, based on the type of surgical intervention, and therefore on the location of the surgical lesion and the organ to be treated, it is possible to use analgesic patterns that involve stimulation (through insertion of needles) of points situated on the meridians that pass through the regions affected by the surgical intervention. In particular, for radical mastectomy surgery, the following points are indicated:

- LU1 or LU2, ST14, ST18, VB24 as proximal points (in the vicinity of the surgical site) KI22, KI27 as points of therapeutic action particularly important in intercostal

- neuralgia.

- ST36 as a distal point (distant from the surgical site) with a general effect

- SP6, MC6 because they have a remarkable antalgic action on the thoracic region.

In our case, we replaced these last two points with LI4 because we preferred to employ the general analgesic power intrinsic to this point.

For the homeopathic treatment we administered to her: Arnica montana $15 \mathrm{CH}$ pills once and Apis mellifica $15 \mathrm{CH}$ pills once.

The morning of the surgery she took 5 pills of Arnica montana $15 \mathrm{CH}$ and underwent a second electro acupuncture session with frequencies of $23-70 \mathrm{~Hz}$ for 20 minutes, repeated after 30 minutes, on the same points as the previous day (Figure 1-2).

\section{CASE 2}

The second patient was a woman 74 years old, weight $75 \mathrm{~kg}$, height $160 \mathrm{~cm}$ with a diagnosis of cutaneous fistulization resulting from a previous local radiotherapy to the left breast.
The pathological anamnesis found non-controlled arterial hypertension, insulin-dependent diabetes mellitus (Type 1) with low control, and a dysmetabolic/exotoxic hepatic cirrhosis. In 1990, she underwent a left quadrantectomy with ipsilateral axillary lymphadenectomy followed by local radiotherapy and chemotherapy for ductal breast cancer.

As in the previous case, we made a hepatic evaluation before the surgery. This revealed hepatic cirrhosis with multifactorial aetiology: dysmetabolism (diabetes poorly controlled for years, overweight) and toxic alcohol-induced (previous abuse, now notably reduced) and a possible minor iatrogenic component (post quadrantectomy chemotherapy in 1990). The patient had a minimal hepatic cytolysis in progress with moderate increase in indicators of cholestasis. To further complicate the situation, we observed signs of compromised hepatic function (Child Pugh class A6), portal hypertension with thrombocytopenia and diffused esophageal varices.

We did not find any contraindication to surgery, but underlined a risk of clinical decompensation and a hepatic functional risk in the post-surgical setting (surgical stress, anesthesiological toxicity...). The chosen surgical procedure for this patient was a complete left simple mastectomy and surgical debridement of the fistulised mammary area. The evening before surgery the patient received Arnica montana $15 \mathrm{CH}$ pills once and Apis mellifica $15 \mathrm{CH}$ pills once. On the day of surgery, the patient underwent to two sessions of electro-acupuncture with frequencies of 23-70 $\mathrm{Hz}$ for 20 minutes, repeated after 30 minutes. The chosen points for this intervention were the following: 1LU - 22 KI - 27 KI - 24 GB - 14 ST - 18 ST - 4 LI - 36 ST. At the same time, the patient took a homeopathic treatment consisting of Arnica montana 15CH 5 pills and Apis mellifica $15 \mathrm{CH}$ 5 pills.

\section{Discussion}

Both of the patients exhibited the following anesthesiological behaviour: before going into the surgical area they took another 5 pills of Arnica montana $15 \mathrm{CH}$ and $2 \mathrm{mg}$ of intravenous midazolam. The latter is a short-acting hypno-inducing drug generally used before and during induction of anaesthesia at a dose of $0.07-0.1 \mathrm{mg} / \mathrm{kg}$. The patients then entered the operating theatre, were placed on the surgical table, and monitored as usual for vital signs: electrocardiogram, heart rate, pulse oximetry, non-invasive measurement of arterial pressure, end-tidal CO2 (Figure 3-4). Based on the general condition of the patients, we chose to use Sevoflurane, an anesthesiological inhalator halogenate metabolized by CYP450 and afterward secreted with urine. Thanks to its low solubility in the blood, this drug achieves a rapid increase in alveolar concentration during induction followed by a rapid decrease when the anaesthesia is discontinued. After induction and curarization with rocuronium, we proceeded to insert the chest tube and the surgeon then continued preparation of the surgical area, as required by the protocol. Both of the patients reached a Minimum Alveolar Concentration (MAC) of $1.4 \%$. The haemodynamical and respiratory parameters recorded during the surgical procedures were steady without use of opioids and/or other pain-relieving 
drugs. The patients remained in the recovery room for a short period of time (about 45 minutes) to evaluate possible onset of AE, pain or complications in the immediate post-surgical period. After this, since no intra-operatory complications or anomalous bleeding were observed, the avoidance of analgesics and the consequent rapid awakening meant the patients did not need to be relocated to a protected area. Instead, they were conducted to their recovery room where they took 5 pills of Arnica montana. The evaluation of the pain on the first day after surgery was very comfortable (Figure 5). The patients were able to drink and eat, and they could get up only a few hours after the end of the surgery. In the evening they took another 5 pills of Arnica montana $15 \mathrm{CH}$. The following morning the patients reported that they slept well, without pain. The hematic values of the liver function test, conducted as a control, were the same as those recorded before surgery. Both of the patients were discharged on the second day post-surgery.

From our findings, we can conclude that electro-acupuncture together with homeopathy is a useful alternative to the common drugs used in both the intra and post-surgery phases. In these practical case studies, we observed that stimulation with needles was able to achieve remarkable stabilization of the autonomic nervous system. Indeed, there were no haemodynamic or blood pressure changes that would have made it necessary to adjust anaesthesia, use vasopressor drugs, deepen hypnosis or administer an opioid or other pain drug. Unlike commonly reported from scientific literature that shows how CAM can help the reduction of analgesic drugs like opioids in intra- and post-surgical phases, we want to show how these medicines can be totally avoided with significative advantages for patients. Indeed, they can have a less number of complications related to drugs and a less work of the excretory organs. In these case studies, the advantage of the alternative treatment was the great support offered to patients, who were thus able to receive fewer drugs associated to numerous potential comorbidities, with an accordingly lower exposure to risk of adverse events. Above all, this treatment avoided the need for post-surgical recovery in a protected area, thereby reducing the hospitalization time. Last but not least, all these new approaches can deliver conspicuous economic savings for the hospital.

The very promising results observed in this study can open new ways for a better and wider use of integrative medicines in surgical settings. It should be emphasized here that these integrative medicines are not intended to replace the conventional treatments. However, as their name indicates, they can provide a significant and useful support in particular cases, such as patients with allergies or organic disorders for whom the common treatments are contra-indicated or might bring many complications. In this way, the application of traditional Chinese medicine can help improve the common practice of Western medicine.

\section{Conflict of interest}

The authors declare that there are no conflicts of interest.

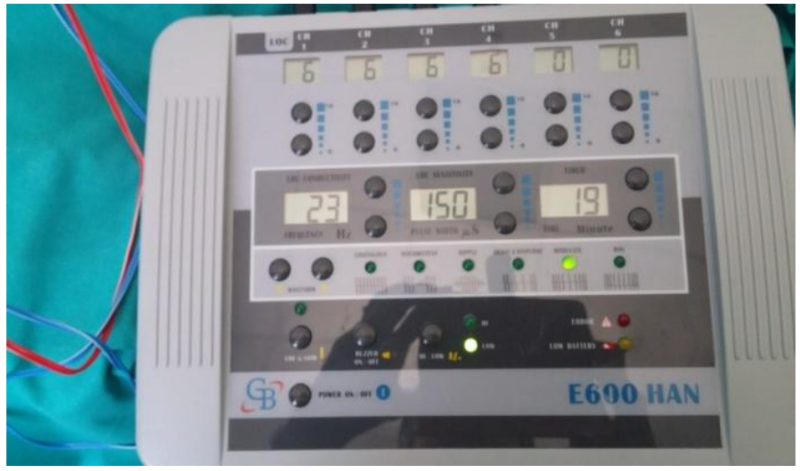

Figure 1 Electro-acupuncture before the surgery

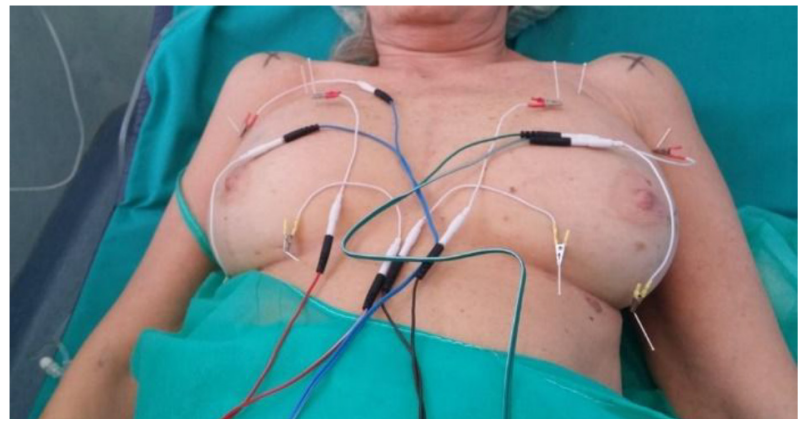

Figure 2 Electro-acupuncture before the surgery

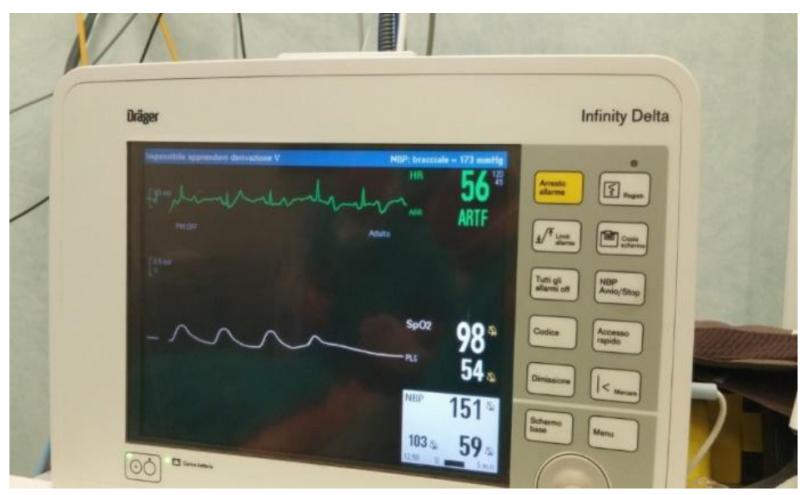

Figure 3 Case 1 - Initial phases of anesthesia

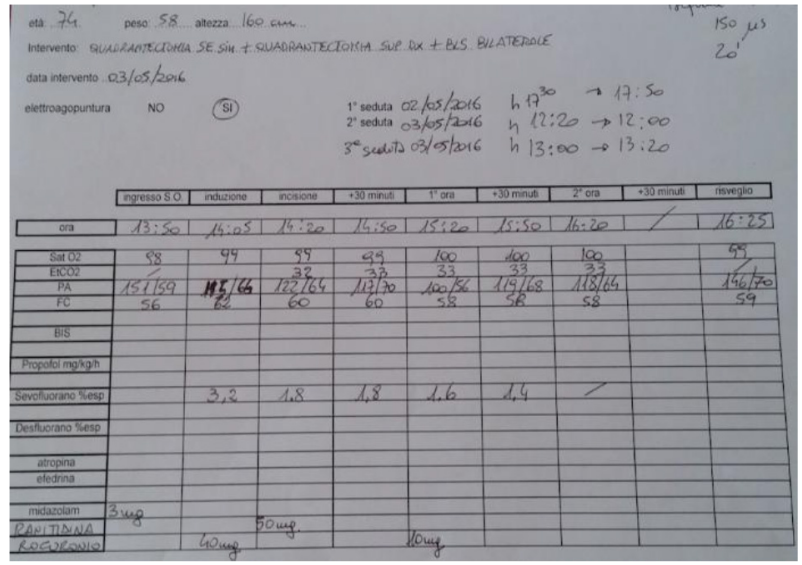

Figure 4 Intra surgical data (Case 1) 


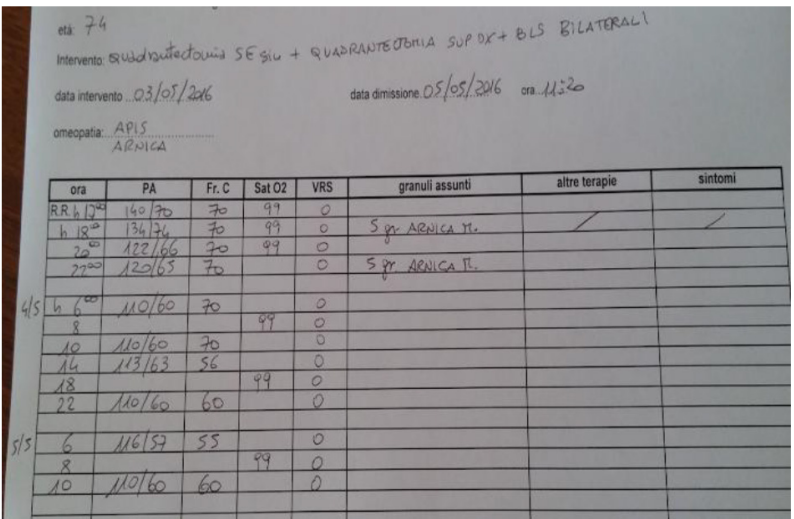

Figure 5 Post-surgical pain evaluation (Case 1) 


\section{References}

1. Kehlet H, Dahl JB. Anaesthesia, surgery, and challenges in postoperative recovery. Lancet. 2003; 362: 1921-8.

2. Chao AS, Chao A, Wang TH, Chang YC, Peng HH, et al. Pain relief by applying transcutaneous electrical nerve stimulation (TENS) on acupuncture points during the first stage of labor: a randomized double-blind placebo-controlled trial. Pain. 2007. 127(3):214-20.

3. Iannitti T, Morales-Medina JC, Bellavite P, Rottigni V, Palmieri B Effectiveness and Safety of Arnica montana in Post-Surgical Setting, Pain and Inflammation. American Journal of Therapeutics. 2014.

4. Robertson A, Suryanarayana N, Banerjee A. Homeopathic Arnica montana for post- tonsillectomy analgesia: a randomized placebo control trial. Homeopathy. 2007.

5. Lao L, Bergman S, Hamilton GR et al. Evaluation of Acupuncture for Pain Control After Oral Surgery: A Placebo-Controlled Trial. Arch Intern Med. 2009; 169 (9), 858.

6. Practice Guidelines for Acute Pain Management in the Perioperative Setting Anesthesiology. 2012; 116 (2).

7. Molassiotis A, Fernadez-Ortega P, Pud D, Ozden G, Scott JA et al. Use of complementary and alternative medicine in cancer patients: a European survey. Annals of Oncology. 2005; 16: 655-663.

8. Bonacchi A, Fazzi L, Toccafondi A, Cantore M, Mambrini A et al. Use and Perceived Benefits of Complementary Therapies by Cancer Patients Receiving Conventional Treatment in Italy. 2013.

9. Rossi E, Vita A, Baccetti S, et al. Complementary and alternative medicine for cancer patients: Results of the EPAAC survey on integrative oncology centres in Europe. Support Care Cancer. 2014.

10. Filshie, J.; Hester, J. Guidelines for providing acupuncture treatment for cancer patients-A peer-reviewed sample policy document. Acupunct. Med. 2006, 24, 172- 182 .

11. Tavares MG et al. Electro-Acupuncture Efficacy on Pain Control after Mandibular Third Molar Surgery. Braz Dent J; 2007. 18(2): 158-162.

12. Van Nghi N., Lanza U., Van Dong M. Teoria e pratica della analgesia con agopuntura. Ed. Alzani; Pinerolo; Torino; 1975.

13. Han JS. Acupuncture: neuropeptide release produced by electrical stimulation of different frequencies. Trends Neurosci. 2003; 26(1):17-22

14. Basbaum AI, Fields HL. Endogenous pain control systems: brainstem spinal pathways and endorphin circuitry. Annu Rev Neurosci. 1984; 7: 309-38.

15. Kawakita K, Okada K. Mechanisms of action of acupuncture for chronic pain relief - polymodal receptors are the key candidates. Acupuncture in medicine. 2006; 24 (Suppl): S58-66.

16. Mayor D. Electroacupuncture: A Practical Manual and Resource. Ed. Livingstone; 2006.

17. Sorrentino L, Piraneo S, Riggio E, et al. Is there a role for homeopathy in breast cancer surgery? A first randomized clinical trial on treatment with Arnica Montana to reduce post-operative seroma and bleeding in patients undergoing total mastectomy. J Intercult Eth- nopharmacol. 2017; 6(1):1e8.

18. Mazzocchi A, Passi L. Retrospective Analysis of 736 implants inserted without antibiotic therapy. J. Oral Maxillofac Surg. 2007; 65: 2321-2323.

19. Wenzel F, Hoegn C, Rox J, Trapp T, Giers G, Fischer J. Homeopathic arnica as supportive therapy in bone marrow donation. Transfus Med Hemother. 2011; 38 (suppl 1):1-72.

20. Berrebi A, Parant O, Ferval F, Thene M, Ayoubi JM, Connan J, Belon P. Traitement de la douleur de la montée laiteuse non souhaitée par homéopathie dans le post-partum immédiat. J Gynecol Obstet Biol Reprod. 2001; 30: 353-357.

21. Merfort I. Arnica: new insights on the molecular mode of action of a traditional medicinal plant; Forsch. Komplementarmed. Klass. Naturheilkd. 2003; 10; 45-48.

22. Lyß G, Knorre A, Schmidt TJ, Pahl HL.The Anti-inflammatory Sesquiterpene Lactone Helenalin Inhibits the Transcription Factor NF-kB by Directly Targeting p65. The Journal of biological chemistry. 1998; 273(50): pp. 33508-33516.

23. Sharma S, Arif M, Nirala RK, Gupta R, Thakur SC. Cumulative therapeutic effects of phytochemicals in Arnica montana flower extract alleviated collagen-induced arthritis: inhibition of both pro-inflammatory mediators and oxidative stress. J Sci Food Agric. 2016; 96: $1500 \pm 1510$.

24. Macedo SB, Ferreira LR, Perazzo FF, Carvalho JC. Anti-inflammatory activity of Arnica montana 6CH: preclinical study in animals. Homeopathy 2004; 93: 84 \pm 87 . PMID: 15139092.

25. Nicollas R, Pondaven S, Giovanni A, Couloigner V, Tronche S, Recommandation pour la pratique Clinique. Société Française d'Anesthésie Réanimation; 2000.

26. Marzotto M, Bonafini C, Olioso D, Baruzzi A, Bettinetti L, Di Leva F, Galbiati E, Bellavite P. Arnica montana Stimulates Extracellular Matrix Gene Expression in a Macrophage Cell Line Differentiated to Wound-Healing Phenotype. Plos one. 2016; 11: e0166340.

27. Bigagli E, Luceri C, Dei A, Bernardini S, Dolara P. Effects of Extreme Dilutions of Apis mellifica Preparations on Gene Expression Profiles of Human Cells. Dose- Response: An International Journal. 2016:1-7 